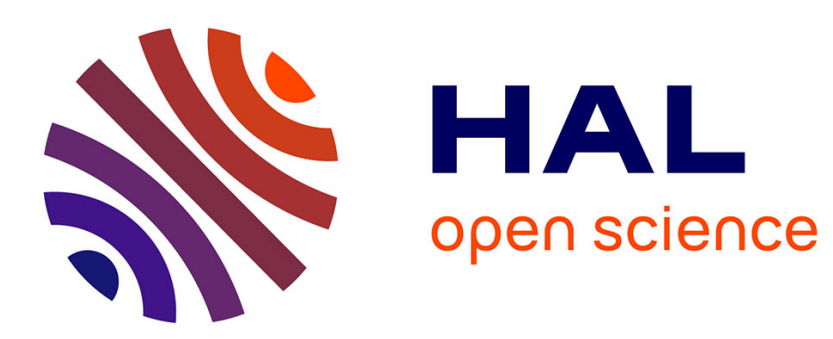

\title{
Attitude tracking control of a flexible spacecraft under angular velocity constraints
}

Laurent Burlion, Jean-Marc Biannic, Tarek Ahmed-Ali

\section{To cite this version:}

Laurent Burlion, Jean-Marc Biannic, Tarek Ahmed-Ali. Attitude tracking control of a flexible spacecraft under angular velocity constraints. International Journal of Control, 2017, 92 (7), pp.1524-1540. 10.1080/00207179.2017.1398841. hal-01664704

\section{HAL Id: hal-01664704 https://hal.science/hal-01664704}

Submitted on 11 Jun 2020

HAL is a multi-disciplinary open access archive for the deposit and dissemination of scientific research documents, whether they are published or not. The documents may come from teaching and research institutions in France or abroad, or from public or private research centers.
L'archive ouverte pluridisciplinaire HAL, est destinée au dépôt et à la diffusion de documents scientifiques de niveau recherche, publiés ou non, émanant des établissements d'enseignement et de recherche français ou étrangers, des laboratoires publics ou privés. 


\title{
Interval based control of a flexible spacecraft under angular velocity constraints
}

\author{
L. Burlion ${ }^{\mathrm{a} *}$, J-M. Biannic ${ }^{\mathrm{a}}$ and T. Ahmed-Ali ${ }^{\mathrm{b}}$ \\ ${ }^{a}$ ONERA, The French Aerospace Lab, F-31055 Toulouse, France; ${ }^{b}$ Laboratoire GREYC, UMR CNRS \\ 6072, Université de Caen Basse-Normandie / ENSICAEN, Caen, France
}

( $v 1.0$ released February 2017)

\begin{abstract}
In this paper, a new technique is proposed for trajectory tracking of a flexible spacecraft subject to angular velocity constraints. The problem is addressed using an Output to Input Saturation Transformation (OIST) which converts the prescribed bounds into state-dependent saturations on the control input signals. It is shown that an interval observer can be used in combination with the OIST technique to ensure that the constraints remain satisfied despite unmeasured flexible modes and torque disturbances. Some realistic simulations conclude the paper and validate our approach.
\end{abstract}

Keywords: constrained control; interval observers; anti-windup; spacecraft; attitude stabilisation; time-domain constraint

\section{Introduction}

State constraints arise in numerous engineering applications. Among several approaches summarized in G.C. Goodwin and de Dona (2004), Glattfelder and Schaufelberger (2003), a popular idea consists of modifying a nominal controller whenever the constraints are about to be violated. Following this line, the Output to Input Saturation Transformation (OIST) approach was recently proposed in Burlion (2012); Chambon, Burlion, and Apkarian (2015a) to replace the state constraints by some state-dependent control saturations. Such an approach is attractive because it enables use of the vast literature on control saturation problems and may especially lead to consider anti-windup loops which are now well understood (see e.g Galeani, Tarbouriech, Turner, and Zaccarian (2009); Tarbouriech and Turner (2009)).

On the other hand, the spacecraft attitude pointing and tracking control problems have been extensively studied in the past decades (see e.g Akella, Thakur, and Mazenc (2015); Chen and Huang (2009); Di Gennaro (2002); Gennaro (2003); H.-H. Zhang and Trivailo. (2008); Lovera and Astolfi (2004); Tayebi (2008); Trégouët, Arzelier, Peaucelle, Pittet, and Zaccarian (2015); Zhang and Cheng (2012) and the references therein). Most of the papers dealing with satellite attitude control subject to constraints use Model Predictive Control (MPC) techniques (see e.g Gupta, Kalabic, Cairano, Bloch, and Kolmanovsky (2015); Hegrenaes, Gravdahl, and Tondel (2005)). Although MPC provides very efficient solutions, the full state is most of the time assumed to be available. As an alternative to MPC, only a few papers deal with state-constraints, especially with the problem of limiting the angular velocity of a spacecraft as in $\mathrm{Hu}$ (2009); Luzi, Peaucelle, Biannic, Pittet, and Mignot (2014). This is a significant problem because the limitations of the satellite actuators require a response with a limited speed when the pointing error is too large.

In this paper, the problem of spacecraft attitude reference trajectory tracking is considered. To cope with angular velocity limitations, a nominal controller is combined with the so-called OIST methodology. Since in its preliminary form, this technique requires the full state to be measured, an 
interval observer is proposed developing the ideas introduced in Chambon, Burlion, and Apkarian (2015b). This observer is used to compute upper and lower bounds of the torque induced by the unmeasured flexible modes and external disturbances. Finally the stability of the closed-loop system is established using an anti-windup design.

The paper is organized as follows. In Section 2, the mathematical model of the flexible spacecraft is recalled, while in Section 3, the control problem is stated. In Section 4, a novel Output to Input Saturation Transformation which uses a time-varying interval observer is proposed to deal with the angular velocity constraints. The obtained closed-loop saturated system is studied in Section 5 : it is demonstrated that asymptotic stability properties are achieved when a nonlinear anti-windup loop is combined with a nominal control law. Finally, the effectiveness of the proposed approach is illustrated by numerical simulations in Section 6 .

\section{Preliminaries}

\subsection{Definitions and notation}

Notation 1: The classical unit quaternion representation for satellite orientation with respect to an inertial frame is denoted $q=\left[q_{0}, q_{v}^{T}\right]^{T}$, where $q_{0} \in \mathcal{R}$ is the scalar component and $q_{v} \in \mathcal{R}^{3}$ is the vector component. The quaternion is unit when $q_{0}^{2}+q_{v}^{T} q_{v}=1$. The inverse of $\mathrm{q}$ is denoted $q^{-1}=\left[q_{0},-q_{v}^{T}\right]^{T}$ and the product of two quaternions $q_{1}$ and $q_{2}$ is denoted $q_{1} * q_{2}$. The following notation will be useful when developing the equations for satellite attitude dynamics:

$$
\begin{gathered}
S(v)=\left[\begin{array}{ccc}
0 & -v_{3} & v_{2} \\
v_{3} & 0 & -v_{1} \\
-v_{2} & v_{1} & 0
\end{array}\right] \quad E(q)=\left[\begin{array}{c}
-q_{v}^{T} \\
q_{0} I_{3}+S\left(q_{v}\right)
\end{array}\right] \\
R(q)=\left(q_{0}^{2}-q_{v}^{T} q_{v}\right) I_{3}+2 q_{v} q_{v}^{T}-2 q_{0} S\left(q_{v}\right)
\end{gathered}
$$

where $v=\left[v_{1}, v_{2}, v_{3}\right]^{T}$. Note that $S($.$) is the skew symmetric operator and that E(q) \in \mathcal{R}^{4 \times 3}$ is classically used in the expression of $\dot{q}$. Moreover, $R(q) \in S O(3)$ is the rotation matrix that corresponds with $q$.

Notation 2: Given two vectors $x_{1}, x_{2} \in \mathbb{R}^{n}$, the relation $x_{1} \leq x_{2}$, is understood element-wise.

Notation 3: For any vector $x \in \mathcal{R}^{n}$, and any matrix $A \in \mathcal{R}^{m \times n},|x|$ and $|A|$ denote respectively the Euclidean norm of $x$ and the induced norm of $A$. The Frobenius norm of is denoted by $|A|_{F}:=$ $\sqrt{\operatorname{trace}\left(A^{T} A\right)}$. Moreover, given any positive definite matrix $Q, \lambda_{\min }(Q)$ (resp. $\lambda_{\max }(Q)$ ) denotes the minimal (resp. maximal) eigenvalue value of $Q$.

Definition 1: A square matrix $M=\left(M_{i j}\right) \in \mathcal{R}^{n \times n}$ is said to be Metzler if $M_{i j} \geq 0, \forall i \neq j$.

Definition 2: Given two scalar functions $\underline{u}, \bar{u}: \mathcal{R} \longrightarrow \mathcal{R}$ such that $\forall t, \underline{u}(t) \leq \bar{u}(t)$, the following saturation operator with time-varying bounds is defined:

$$
\forall u \in \mathcal{R}, \quad S_{a} t_{\underline{u}(t)}^{\bar{u}(t)}(u)=\max \{\underline{u}(t), \min \{\bar{u}(t), u\}\}
$$

When applied to a vector, this operator is understood component-wise.

\section{$2.2 \quad$ Flexible spacecraft modeling}

In this paper, we consider the flexible spacecraft model used in Di Gennaro (2002): 


$$
\begin{aligned}
\dot{q}_{e} & =\frac{1}{2} E\left(q_{e}\right) \omega_{e} \\
J_{m b} \dot{\omega}_{e} & =-N\left(\omega, \omega_{e}, z_{e}, \omega_{r}^{b}\right)+u+d+C_{z} z_{e}+D_{z} \omega_{e}-J_{m b} \dot{\omega}_{r}^{b} \\
\dot{z}_{e} & =A_{z} z_{e}+B_{1, z} \omega_{e}+B_{2, z} \dot{\omega}_{r}^{b}
\end{aligned}
$$

where:

- $q_{e}=q * q_{r}^{-1}=\left[\begin{array}{ll}q_{e 0} & q_{e v}^{T}\end{array}\right]^{T}$ is the error between the spacecraft quaternion $q$ and the reference quaternion $q_{r}$

- $\omega_{e}=\omega-\omega_{r}^{b} \in \mathcal{R}^{3}$ is the error between the angular velocity of the spacecraft $\omega$ and $\omega_{r}^{b}$ which is the reference angular velocity $\omega_{r}$ expressed in the body fixed frame. In other words, $\omega_{r}^{b}$ is defined by:

$$
\omega_{r}^{b}=R\left(q_{e}\right) \omega_{r}=R(q) R\left(q_{r}\right)^{T} \omega_{r}
$$

- $u \in \mathcal{R}^{3}$ are the control inputs produced by gas jets.

- $d \in \mathcal{R}^{3}$ are the torque disturbances.

- $z_{e}=z-z_{r}$ where $z=[\eta ; \psi]=[\eta ; \dot{\eta}+\delta \omega]\left(\eta \in \mathcal{R}^{N}\right.$ being the vector of the modal displacements) and $z_{r}:=\left[O_{N, 1} ; \delta \omega_{r}^{b}\right]$.

- $J_{m b}=J-\delta^{T} \delta$ is the main body symmetric inertia matrix where $J \in \mathcal{R}^{3 \times 3}$ is the symmetric inertia matrix of the undeformed structure and $\delta \in \mathcal{R}^{N \times 3}$ is the coupling matrix between elastic and rigid dynamics

- $\left(A_{z}, B_{1, z}, B_{2, z}, C_{z}, D_{z}\right)$ are matrices expressed with respect to the damping (resp. stiffness) matrix $C=\operatorname{diag}\left\{2 \xi_{i} \omega_{i}, i=1 . . N\right\}$ (resp. $\left.K=\operatorname{diag}\left\{\omega_{i}^{2}, i=1 . . N\right\}\right)$ of the $N$ flexible modes:

$$
\begin{aligned}
& A_{z}=\left[\begin{array}{cc}
O_{N} & I_{N} \\
-K & -C
\end{array}\right] \quad, \quad B_{2, z}=-\left[\begin{array}{c}
O_{N} \\
I_{N}
\end{array}\right] \delta \quad, \quad B_{1, z}=A_{z} B_{2, z} \\
& C_{z}=\delta^{T}[K C] \quad, \quad D_{z}=-\delta^{T} C \delta
\end{aligned}
$$

- the function $N$ is defined by the following relation:

$$
\forall e, f, g \in \mathcal{R}^{3}, \forall z \in \mathcal{R}^{2 N}, N(e, f, z, h)=S(e)\left(J_{m b} f+G_{z} z+J h\right)
$$

where $G_{z}=\left[\begin{array}{ll}O_{3, N} & \delta^{T}\end{array}\right]$

Remark 1: The expression retained for $z_{r}$ corresponds to the absence of modal displacements. Indeed, $z_{r}$ can be rewritten as $z_{r}:=\left[\eta_{r} ; \dot{\eta}_{r}+\delta \omega_{r}^{b}\right]$ where $\eta_{r}=\dot{\eta}_{r}=O_{N, 1}$. Moreover, considering the expressions of $A_{z}, B_{1, z}$ and $B_{2, z}(5)$, it can be easily shown that $z_{r}$ verifies $\dot{z}_{r}=A_{z} z_{r}+B_{1, z} \omega_{r}^{b}-$ $B_{2, z} \dot{\omega}_{r}^{b}$.

Remark 2: Control inputs $u$ are merely produced by gas jets in the retained model of Di Gennaro (2002). Considering additional control inputs like reaction wheels and magnetorquers is postponed to future studies

\section{Problem formulation}

\subsection{Main assumptions}

In the remainder of the paper, the following not so restrictive assumptions have been made: 
(A1) Although it might be poorly damped, the spacecraft structure is assumed strictly stable so that $A_{z}$ is Hurwitz (all eigenvalues lie strictly inside the open left half plane),

(A2) The torque disturbance signal is bounded. More precisely, we assume that:

$$
d \in \mathcal{L}_{2} \cap \mathcal{L}_{\infty}
$$

which implies:

$$
\exists \bar{d}>0 / d(t) \in[-\bar{d}, \bar{d}]
$$

(A3) The initial state vector $z(0) \in \mathbb{R}^{2 N}$ associated to the flexible modes is assumed to belong to a bounded interval. Then, there exist $z_{0}^{-}, z_{0}^{+} \in \mathbb{R}^{2 N}$ such that $z_{0}^{-} \leq z(0) \leq z_{0}^{+}$or equivalently:

$$
z(0) \in\left[z_{0}^{-}, z_{0}^{+}\right]
$$

(A4) The reference angular velocity $\omega_{r}$ and the acceleration $\dot{\omega}_{r}$ are assumed to be bounded. More precisely, we will consider:

$$
\omega_{r} \in \mathcal{L}_{\infty} \quad ; \quad \dot{\omega}_{r} \in \mathcal{L}_{2} \cap \mathcal{L}_{\infty}
$$

\subsection{Problem statement}

The starting point of the paper is the following nominal control law which does not take into account the angular velocity constraints and requires the whole state to be measured:

Lemma 1: Under assumptions (A2) and (A4) and further considering that the whole state is measurable, let the following static state-feedback controller be applied to system (3a)-(3c)

$$
\begin{aligned}
u=u_{n o m}\left(q_{e 0}, x_{e}, \omega_{r}^{b}, \dot{\omega}_{r}^{b}\right)= & -k_{p} q_{e v}-k_{d} \omega_{e}-\frac{1}{2} J_{m b}\left(q_{e 0} I_{3}+S\left(q_{e v}\right)\right) \omega_{e}+N\left(\omega, \omega_{e}, z_{e}, \omega_{r}^{b}\right) \\
& -C_{z} z_{e}-D_{z} \omega_{e}+J_{m b} \dot{\omega}_{r}^{b}
\end{aligned}
$$

where the controller gains $k_{p}$ and $k_{d}$ are strictly positive scalars. Then, the error state $x_{e}=$ $\left[q_{e v} ; \omega_{e} ; z_{e}\right]$ asymptotically converges to the origin for any initial condition $x(0)$.

Proof. The control law is similar to the one of [Theorem 1, Di Gennaro (2002)]. There are two slight differences in the proof of the result:

(1) in our modeling, we consider a torque disturbance $d$ which introduces an additional term in the derivative of the Lyapunov function $V$. This is treated in the same manner that the $\dot{\omega}_{r}$ term. Indeed, using assumption (A2), one can still conclude the proof by invoking Barbalat's lemma.

(2) we do not impose any lower-bound on $k_{d}$ since the proof is unchanged when $P$ is replaced by $\varepsilon P$ where the positive real $\varepsilon$ is an extra degree of freedom which can be chosen sufficiently small so that $Q>0$ no matter the value of $k_{d}>0$.

Remark 3: Note that $q_{e v}$ tends to 0 while $q_{e_{0}}$ is driven to \pm 1 which in all cases make $R\left(q_{e}\right)$ converge to the same rotation $I_{3}$. Akella et al. (2015) proposes to adopt the notion of 'almost' global asymptotic stability for this problem. 
In this paper, the problem at hand is thus to redesign the nominal full state feedback control law (11) in the case where

- the modal displacements $\eta$ and their time derivatives $\dot{\eta}$ are not measured, which means that $z$ is no longer available for feedback,

- the angular velocity vector $\omega$ must satisfy the following asymmetric constraints

$$
\forall t, \quad \underline{\omega} \leq \omega(t) \leq \bar{\omega}
$$

with

$$
\underline{\omega}<0<\bar{\omega}
$$

\section{Output to Input Saturation Transformation extended for Robustness (OISTeR)}

Before stating our main results and for clarity of presentation, we first recall the OIST technique when both the full state and the disturbance are measured. Then, we propose an extended technique using the upper and lower bounds of the unmeasured states which are thus provided by the interval observer. This new technique called OISTeR means OIST extended with Robustness properties (with respect to both the uncertain initial condition $z(0) \in\left[z_{0}^{-}, z_{0}^{+}\right]$and uncertainties on $d \in$ $[-\bar{d}, \bar{d}])$.

\subsection{The 'classical' OIST methodology}

For clarity of presentation, let us first recall the OIST methodology when the full state and the disturbance $d$ are measured.

Lemma 2: Let $k_{O}$ be a strictly positive real number. Let $u \in \mathcal{L}_{2}$. Suppose that the whole state and the disturbance torque $d$ are available for feedback. Suppose that $\omega(0) \in[\underline{\omega}, \bar{\omega}]$ and consider the following input saturated system:

$$
\begin{aligned}
J_{m b} \dot{\omega} & =-N(\omega, \omega, z, 0)+O I S a t(u)+d+C_{z} z+D_{z} \omega \\
\dot{z} & =A_{z} z+B_{1, z} \omega
\end{aligned}
$$

where:

$$
\operatorname{OISat}(u):=J_{m b}\left(\operatorname{Sat}_{k_{O} \underline{\omega}}^{k_{o} \bar{\omega}}\left(\alpha+k_{O} \omega\right)-k_{O} \omega\right)+N(\omega, \omega, z, 0)-d-C_{z} z-D_{z} \omega
$$

with:

$$
\alpha=J_{m b}^{-1}\left(-N(\omega, \omega, z, 0)+u+d+C_{z} z+D_{z} \omega\right)
$$

Then, $\forall t \geq 0, \omega(t) \in[\underline{\omega}, \bar{\omega}]$

Proof. The proof is straightforward using the OIST technique as defined in Burlion (2012); Chambon et al. (2015a) in the relative degree 1 case (i.e when $u$ appears in the expression of the first derivative of $\omega$ with respect to time). For clarity, we develop the basic computations. Using (14a)$(15)$, it is readily seen that $\omega$ undergoes the following dynamics:

$$
\dot{\omega}=S_{a t}^{k_{O} \underline{\omega}}\left(\alpha+k_{O} \omega\right)-k_{O} \omega
$$


which implies that $\forall t$,

$$
-k_{O}(\omega-\underline{\omega}) \leq \dot{\omega} \leq-k_{O}(\omega-\bar{\omega})
$$

from this, the result is easily deduced using the fact that $\omega(0) \in[\underline{\omega}, \bar{\omega}]$ and $k_{O}>0$.

Remark 4: 'OISat $(\mathrm{u})$ ' denotes a saturation of the control input $u$. This notation is preferred to 'Sat' since it is a state dependent saturation element which comes from an Output to Input Saturation transformation.

\subsection{The OISTeR methodology}

The OISTeR methodology is now developed : it extends the above result when neither $z$ nor $d$ are no longer measured. Following the preliminary ideas of Chambon et al. (2015b), we first build an interval observer in order to obtain lower and upper bounds of the missing variables. Then, we propose a novel 'OISat' function which exploits these bounds. Note that the use of an interval observer is the key idea to respect the angular velocity constraints (using an estimation of $z$ in combination with the OIST methodology presented in Lemma 1 would not be sufficient to make sure that these constraints are fulfilled).

\subsubsection{Interval observer synthesis}

Let us introduce the following quantity:

$$
\begin{aligned}
\dot{\hat{z}} & =A_{z} \hat{z}+B_{1, z} \omega \\
\hat{z}(0) & =\frac{z_{0}^{+}+z_{0}^{-}}{2}
\end{aligned}
$$

Let us denote $e_{z}=z-\hat{z}$ and consider the following system:

$$
\begin{aligned}
& \dot{e}_{z}=A_{z} e_{z} \\
& e_{y}=J_{m b}^{-1}\left(-S(\omega) G_{z} e_{z}+C_{z} e_{z}+d\right)
\end{aligned}
$$

Lemma 3: Let the damping ratios verify $0<\xi_{i}<1$ for all $i \in\{1, N\}$. Then, under assumptions (A2) and (A3), there exist a Hurwitz Metzler matrix $M \in \mathcal{R}^{2 N \times 2 N}, Q_{0} \in \mathcal{R}^{2 N \times 2 N}$ and an operator $\Pi: \mathcal{R}^{4} \longrightarrow \mathcal{R}^{2 N \times 2 N}$ such that:

$$
\forall t \geq 0,\left|e_{y}\right| \leq \bar{e}_{y}
$$

with

$$
\begin{aligned}
\bar{e}_{y} & =\Pi(t, \omega) \zeta^{+}+\left|J_{m b}^{-1}\right| \bar{d} \\
\dot{\zeta}^{+} & =M \zeta^{+} \\
\zeta(0) & =\left|Q_{0}\right| \frac{z_{0}^{+}-z_{0}^{-}}{2}
\end{aligned}
$$

Proof. the proof of this result is reported in Appendix A. 
Remark 5: As evidenced by the proof, the result is obtained by using a time-varying interval observer for system (20a). Such an observer is necessarily chosen here since the state matrix $A_{z}$ exhibits a poor damping. Let us indeed rewrite the latter in the appropriate basis such that $A_{z} \sim \operatorname{blkdiag}\left(J_{i}, i=1 . . N\right)$ with

$$
J_{i}=\left[\begin{array}{cc}
0 & 1 \\
-\omega_{i}^{2} & -2 \xi_{i} \omega_{i}
\end{array}\right]
$$

Next, compute the following key mapping

$$
\mathcal{F}\left(J_{i}\right)=\operatorname{tr}\left(J_{i}\right)^{2}-2 \operatorname{det}\left(J_{i}\right)=4 \xi_{i}^{2} \omega_{i}^{2}-2 \omega_{i}^{4}
$$

As a consequence of [Thm 2,Mazenc and Bernard (2010)], when the following condition (25) holds,

$$
\mathcal{F}\left(J_{i}\right)<0 \Leftrightarrow \xi_{i}<\frac{\sqrt{2}}{2} \omega_{i}
$$

there does not exist any linear time-invariant change of coordinates transforming $\dot{N}_{i}=J_{i} N_{i}$ to a system for which a 'classical' exponentially stable framer can be used. In that case which usually corresponds to poorly damped systems - thus involving flexible modes - Mazenc and Bernard (2010) suggests to use a time-varying interval observer.

\subsubsection{Design of a novel 'OISat' function}

Lemma 4: Let $k_{O}$ be a strictly positive real number. Let $d \in \mathcal{L}_{2}$. Let assumptions (A2) and (A3) hold and suppose that $\omega(0) \in[\underline{\omega}, \bar{\omega}]$. Consider the input saturated system (14a)-(14b) with:

$$
\operatorname{OISat}(u):=J_{m b}\left(\operatorname{Sat}_{k(t) \underline{\omega}+\bar{e}_{y}}^{k(t) \overline{e_{y}}}(\alpha+k(t) \omega)-k(t) \omega\right)+N(\omega, \omega, \hat{z}, 0)-C_{z} \hat{z}-D_{z} \omega
$$

and:

$$
\begin{aligned}
\alpha & =J_{m b}^{-1}\left(-N(\omega, \omega, \hat{z}, 0)+u+C_{z} \hat{z}+D_{z} \omega\right) \\
k(t) & =k_{O}+\frac{2 \bar{e}_{y}}{\bar{\omega}-\underline{\omega}} \\
\dot{\hat{z}} & =A_{z} \hat{z}+B_{1, z} \omega \\
\hat{z}(0) & =\frac{z_{0}^{-}+z_{0}^{+}}{2}
\end{aligned}
$$

Then,

$$
\forall t \geq 0, \quad \omega(t) \in[\underline{\omega}, \bar{\omega}]
$$

Proof. Using (14a)-(14b)-(20b)-(44b)-(19a), it is readily checked that $\omega$ undergoes the following dynamics:

$$
\dot{\omega}=S a t_{k(t) \underline{\omega}+\bar{e}_{y}}^{k(t) \overline{-}-\bar{e}_{y}}(\alpha+k(t) \omega)-k(t) \omega+e_{y}
$$

Note that the saturation function is well defined because (27b) implies that,

$$
\forall t \geq 0, \quad k(t) \bar{\omega}-\bar{e}_{y} \geq k(t) \underline{\omega}+\bar{e}_{y}
$$


it follows from (29) that

$$
\forall t \geq 0, \quad-k(t)(\omega-\underline{\omega})+e_{y}+\bar{e}_{y} \leq \dot{\omega} \leq e_{y}-\bar{e}_{y}-k(t)(\omega-\bar{\omega})
$$

which implies that

$$
\forall t \geq 0, \quad-k(t)(\omega-\underline{\omega}) \leq \dot{\omega} \leq-k(t)(\omega-\bar{\omega})
$$

Recalling that $\omega(0) \in[\underline{\omega}, \bar{\omega}]$ and noting from $(13,27 \mathrm{~b})$ that $k(t) \geq k_{O}>0$, the final result (28) immediately follows from (32) which concludes the proof.

\section{Main result : attitude tracking under velocity constraints}

Based on the notation introduced in subsection 4.2.1 dedicated to interval observer design, and more specifically on equations (19a)-(20b), the flexible spacecraft model (3a)-(3c) may be rewritten as follows:

$$
\begin{aligned}
\dot{q}_{e} & =\frac{1}{2} E\left(q_{e}\right) \omega_{e} \\
J_{m b} \dot{\omega}_{e} & =-N\left(\omega, \omega_{e}, \hat{z}_{e}, \omega_{r}^{b}\right)+u+C_{z} \hat{z}_{e}+D_{z} \omega_{e}+J_{m b}\left(e_{y}-\dot{\omega}_{r}^{b}\right) \\
\dot{\hat{z}}_{e} & =A_{z} \hat{z}_{e}+B_{1, z} \omega_{e}+B_{2, z} \dot{\omega}_{r}^{b} \\
\dot{e}_{z} & =A_{z} e_{z}
\end{aligned}
$$

with

$$
\begin{aligned}
\hat{z}_{e} & =\hat{z}-z_{r} \\
e_{z} & =z-\hat{z} \\
e_{y} & =J_{m b}^{-1}\left(-S(\omega) G_{z} e_{z}+C_{z} e_{z}+d\right)
\end{aligned}
$$

and the main result of the paper, summarized in the following theorem, may now be stated.

Theorem 1: Let $k_{O}, k_{a}$ be strictly positive real numbers. Let $d \in \mathcal{L}_{2}$. Let assumptions (A2) to (A4) hold and suppose that $\omega(0) \in[\underline{\omega}, \bar{\omega}]$. Consider a closed-loop system consisting of the plant (33a)-(33d) and the dynamic control law:

$$
\begin{aligned}
u= & \operatorname{OISat}\left(\tilde{u}_{n}+u_{a}\right) \\
= & J_{m b}\left(\operatorname{Sat}_{k(t) \underline{\omega} \underline{\omega}+\bar{e}_{y}}^{k(t) \overline{-}}(\alpha+k(t) \omega)-k(t) \omega\right)+N(\omega, \omega, \hat{z}, 0)-C_{z} \hat{z}-D_{z} \omega \\
\dot{q}_{a}= & \frac{1}{2} E\left(q_{a}\right) \omega_{a}:=\frac{1}{2} E\left(q_{a}\right) R\left(q_{e}\right) R\left(q_{a}\right)^{T} \omega_{a}^{b e} \\
J_{m b} \dot{\omega}_{a}^{b e}= & \left(N\left(\tilde{\omega}+\omega_{r}^{b}, \tilde{\omega}, \hat{\tilde{z}}, \omega_{r}^{b}\right)-N\left(\omega, \omega_{e}, \hat{z}_{e}, \omega_{r}^{b}\right)\right)+C_{z} z_{a}+D_{z} \omega_{a}^{b e} \\
& +\operatorname{OISat}\left(\tilde{u}_{n}+u_{a}\right)-\tilde{u}_{n} \\
\dot{z}_{a}= & A_{z} z_{a}+B_{1, z} \omega_{a}^{b e}
\end{aligned}
$$


with:

$$
\begin{aligned}
\tilde{u}_{n} & =u_{n o m}\left(\tilde{q}_{0}, \hat{\tilde{x}}, \omega_{r}^{b}, \dot{\omega}_{r}^{b}\right) \\
\alpha & =J_{m b}^{-1}\left(-N(\omega, \omega, \hat{z}, 0)+\tilde{u}_{n}+u_{a}+C_{z} \hat{z}+D_{z} \omega\right) \\
u_{a} & =J_{m b}\left(-k_{a} q_{a v}-k(t) \omega_{a}^{b e}\right)+N\left(\omega, \omega_{a}^{b e}, z_{a}, 0\right)-C_{z} z_{a}-D_{z} \omega_{a}^{b e}
\end{aligned}
$$

where

- $\hat{\tilde{z}}=\hat{z}_{e}-z_{a}, \tilde{\omega}=\omega_{e}-\omega_{a}^{b e}, \tilde{q}=q_{e} * q_{a}^{-1}$.

- $\bar{e}_{y}($ resp.k(t)) is given by (22a) (resp. (27b)).

Then, $\forall t \geq 0, \omega(t) \in[\underline{\omega}, \bar{\omega}]$ and there exists $\underline{k}_{O}$ such that the error $x_{e}=\left[q_{e v} ; \omega_{e} ; z_{e}\right]$ is asymptotically stabilized to the origin for $k_{O} \geq \underline{k_{O}}$.

Remark 6: Note that the dynamic part of the control law described by equations (39)-(42), is the anti-windup loop.

Proof. The proof of the result is divided into two parts. In the first part, it is shown that the error system whose state $\tilde{x}=\left[\tilde{q}_{v} ; \tilde{\omega} ; \tilde{z}\right]$ asymptotically converges to 0 . The second part of the proof establishes that the anti-windup state denoted by $x_{a}=\left[q_{a v} ; \omega_{a}^{b e} ; z_{a}\right]$ asymptotically converges to the origin as well. As a consequence, the state of the error system $x_{e}=\left[q_{e v} ; \omega_{e} ; z_{e}\right]$ tends asymptotically to 0.

Part 1. Let us consider the error system whose state is $\tilde{x}$. After straightforward computations, one obtains:

$$
\left\{\begin{aligned}
\dot{\tilde{q}} & =\frac{1}{2} E(\tilde{q}) \tilde{\omega} \\
J_{m b} \dot{\tilde{\omega}} & =-N\left(\tilde{\omega}+\omega_{r}^{b}, \tilde{\omega}, \hat{\tilde{z}}, \omega_{r}^{b}\right)+u_{n o m}\left(\tilde{q} 0, \hat{\tilde{x}}, \omega_{r}^{b}, \dot{\omega}_{r}^{b}\right)+C_{z} \tilde{z}+D_{z} \tilde{\omega}+J_{m b}\left(e_{y}-\dot{\omega}_{r}^{b}\right) \\
\dot{\tilde{z}} & =A_{z} \tilde{z}+B_{1, z} \tilde{\omega}+B_{2, z} \dot{\omega}_{r}^{b}
\end{aligned}\right.
$$

Following the ideas of the proof of [Theorem 1,Di Gennaro (2002)], we first consider the following Lyapunov function:

$$
V(t, \tilde{x})=\left(k_{p}+k_{d}\right)\left[\left(1-\tilde{q}_{0}\right)^{2}+\left|\tilde{q}_{v}\right|^{2}\right]+\frac{1}{2}\left(\tilde{q}_{v}+\tilde{\omega}\right)^{T} J_{m b}\left(\tilde{q}_{v}+\tilde{\omega}\right)+\frac{\varepsilon}{2} \tilde{z}^{T} P_{z} \tilde{z}+\gamma e_{z}^{T} P_{z} e_{z}
$$

where $\varepsilon, \gamma>0$ and $P_{z}=P_{z}^{T}>0$ is a solution to:

$$
P_{z} A_{z}+A_{z}^{T} P_{z}=-2 Q_{z}
$$

for any fixed $Q_{z}=Q_{z}^{T}>0$. ( $P_{z}$ exists because $A_{z}$ is strictly Hurwitz stable).

Computing the derivative of $V$, one gets:

$$
\dot{V}(t, \tilde{x})=-\tilde{x}^{T} Q \tilde{x}-\gamma e_{z}^{T} Q_{z} e_{z}+\left(\tilde{q}_{v}+\tilde{\omega}\right)^{T}\left(e_{y}-\dot{\omega}_{r}^{b}\right)
$$

with

$$
Q=\left[\begin{array}{ccc}
k_{p} I_{3} & O & O \\
O & k_{d} I_{3} & \frac{\varepsilon}{2} B_{1, z}^{T} P_{z} \\
O & \frac{\varepsilon}{2} P_{z} B_{1, z} & \varepsilon Q_{z}
\end{array}\right]
$$

which, for sufficiently small $\varepsilon>0$, is positive definite. 
Then, using (36) and the boundedness of $\omega$ (which follows from (38) and Lemma (3)), there exist $\lambda_{1}, \lambda_{2}, \lambda_{3}>0$ such that:

$$
\dot{V}(t, \tilde{x})=-\lambda_{\min }(Q)|\tilde{x}|^{2}-\gamma e_{z}^{T} Q_{z} e_{z}+\lambda_{1}|\tilde{x}|\left|e_{z}\right|+\lambda_{2}|\tilde{x}||d|+\lambda_{3}|\tilde{x}|\left|\dot{\omega}_{r}\right|
$$

which, for $\gamma>0$ chosen sufficiently large, yields:

$$
\dot{V}(t, \tilde{x}) \leq-\frac{\lambda_{\min }(Q)}{2}|\tilde{x}|^{2}-\frac{\gamma}{2} e_{z}^{T} Q_{z} e_{z}+\lambda_{2}|\tilde{x}||d|+\lambda_{3}|\tilde{x}|\left|\dot{\omega}_{r}\right|
$$

where $d, \dot{\omega}_{r} \in \mathcal{L}_{2}$ in virtue of Assumptions (A2) and (A4). The proof of this part ends by invoking Barbalat's Lemma as in the proof of [Theorem 1,Di Gennaro (2002)].

Remark 7: As a result, $\tilde{x}$ is uniformly bounded. Furthermore, $\omega$ being bounded implies that $\omega_{a}^{b}:=\omega-\tilde{\omega}-\omega_{r}^{b a}$ is bounded. From Assumption (A1) and equation (42), it readily follows that $z_{a}$ is bounded. Moreover, $q_{a}$ being a unit quaternion, $q_{a v}$ is bounded. As a consequence, there exist well defined positive constants $\tilde{c}, c_{a}$ such that :

$$
\forall t, \quad|\tilde{x}(t)| \leq \tilde{c} \quad\left|x_{a}(t)\right| \leq c_{a}
$$

\section{Part 2.}

On the other hand, the nonlinear anti-windup loop (39)-(42) is now rewritten as follows:

$$
\left\{\begin{aligned}
\dot{q}_{a} & =\frac{1}{2} E\left(q_{a}\right)\left(\omega_{a}^{b e}+\Delta_{q}\right) \\
J_{m b} \dot{\omega}_{a}^{b e} & =\left(-N\left(\omega, \omega_{a}^{b e}, z_{a}, 0\right)+C_{z} z_{a}+D_{z} \omega_{a}^{b e}\right)+\operatorname{OISat}\left(u_{a}\right)+\Delta_{\omega} \\
\dot{z}_{a} & =A_{z} z_{a}+B_{1, z} \omega_{a}^{b e}
\end{aligned}\right.
$$

where:

$$
\begin{aligned}
\Delta_{q}= & \left(R(\tilde{q})^{T}-I_{3}\right) \omega_{a}^{b e} \\
\Delta_{\omega}= & \operatorname{OISat}\left(u_{a}+\tilde{u}_{n}\right)-\operatorname{OISat}\left(u_{a}\right)-\tilde{u}_{n}+N\left(\tilde{\omega}+\omega_{r}^{b}, \tilde{\omega}, \hat{\tilde{z}}, \omega_{r}^{b}\right) \\
& +\left(N\left(\omega, \omega_{a}^{b e}, z_{a}, 0\right)-N\left(\omega, \omega_{e}, \hat{z}_{e}, \omega_{r}^{b}\right)\right) \\
\operatorname{OISat}\left(u_{a}\right)= & J_{m b}\left(\operatorname{Sat}_{k(t) \underline{\omega}+\bar{e}_{y}}^{k(t) \overline{e_{y}}}\left(\alpha_{a}+k(t) \omega_{a}^{b e}\right)-k(t) \omega_{a}^{b e}\right) \\
& +N\left(\omega, \omega_{a}^{b e}, z_{a}, 0\right)-C_{z} z_{a}-D_{z} \omega_{a}^{b e} \\
\alpha_{a}= & J_{m b}^{-1}\left(-N\left(\omega, \omega_{a}^{b e}, z_{a}, 0\right)+u_{a}+C_{z} z_{a}+D_{z} \omega_{a}^{b e}\right)
\end{aligned}
$$

Substituting (58) in (53) results in the following subsystem:

$$
\left\{\begin{aligned}
\dot{q}_{a} & =\frac{1}{2} E\left(q_{a}\right)\left(\omega_{a}^{b e}+\Delta_{q}\right) \\
J_{m b} \dot{\omega}_{a}^{b e} & =J_{m b}\left(S a t_{k(t) \underline{\omega}+\bar{e}_{y}}^{k(t) \overline{e_{y}}}\left(\alpha_{a}+k(t) \omega_{a}^{b e}\right)-k(t) \omega_{a}^{b e}\right)+\Delta_{\omega} \\
\dot{z}_{a} & =A_{z} z_{a}+B_{1, z} \omega_{a}^{b e}
\end{aligned}\right.
$$


Let us now apply the control law $u_{a}$ defined by (44c). It readily follows from (59) and (60) that:

$$
\left\{\begin{aligned}
\dot{q}_{a} & =\frac{1}{2} E\left(q_{a}\right)\left(\omega_{a}^{b e}+\Delta_{q}\right) \\
J_{m b} \dot{\omega}_{a}^{b e} & =J_{m b}\left(S a t_{k(t) \underline{\omega}-\bar{e}_{y}}^{k(t) \bar{e}}\left(-k_{a} q_{a v}\right)-k(t) \omega_{a}^{b e}\right)+\Delta_{\omega} \\
\dot{z}_{a} & =A_{z} z_{a}+B_{1, z} \omega_{a}^{b e}
\end{aligned}\right.
$$

Now considering the time-varying bounds of the saturation operator and substitute $k(t)$ as in (27b), one obtains:

$$
\left\{\begin{array}{l}
k(t) \bar{\omega}-\bar{e}_{y}=k_{O} \bar{\omega}+\frac{\bar{\omega}+\underline{\omega}}{\bar{\omega}-\underline{\omega}} \bar{e}_{y} \\
k(t) \underline{\omega}+\bar{e}_{y}=k_{O \underline{\omega}}+\frac{\bar{\omega}+\underline{\omega}}{\bar{\omega}-\underline{\omega}} \bar{e}_{y}
\end{array}\right.
$$

From (22a)-(22b), it readily follows that $\tilde{\bar{y}}$ tends asymptotically to $\left|J_{m b}^{-1}\right| \bar{d}$. Thus there exists $T \geq 0$ such that :

$$
\forall t \geq T,\left|J_{m b}^{-1}\right| \bar{d} \leq \bar{e}_{y} \leq 2\left|J_{m b}^{-1}\right| \bar{d}
$$

which together with (62) implies that:

$$
\forall t \geq T,\left\{\begin{array}{l}
k(t) \bar{\omega}-\bar{e}_{y} \geq k_{O} \bar{\omega}+\frac{\bar{\omega}+\underline{\omega}}{\bar{\omega}-\underline{\omega}}\left|J_{m b}^{-1}\right| \bar{d} \\
k(t) \underline{\omega}+\bar{e}_{y} \leq k_{O} \underline{\omega}+2 \frac{\bar{\omega}+\underline{\omega}}{\bar{\omega}-\underline{\omega}}\left|J_{m b}^{-1}\right| \bar{d}
\end{array}\right.
$$

Thus, choosing

$$
k_{O}>\max \left\{0, \frac{1}{\bar{\omega}}\left(k_{a}-\frac{\bar{\omega}+\underline{\omega}}{\bar{\omega}-\underline{\omega}}\left|J_{m b}^{-1}\right| \bar{d}\right), \frac{-1}{\underline{\omega}}\left(k_{a}+2 \frac{\bar{\omega}+\underline{\omega}}{\bar{\omega}-\underline{\omega}}\left|J_{m b}^{-1}\right| \bar{d}\right)\right\}
$$

one gets:

$$
\forall t \geq T,\left\{\begin{array}{l}
k(t) \bar{\omega}-\bar{e}_{y} \geq k_{a} \\
k(t) \underline{\omega}+\bar{e}_{y} \leq-k_{a}
\end{array}\right.
$$

As a consequence,

$$
\forall t \geq T, S a t_{k(t) \underline{\omega}+\bar{e}_{y}}^{k(t) \overline{e_{y}}}\left(-k_{a} q_{a v}\right)=-k_{a} q_{a v}
$$

Hence, the solutions being bounded for all time in virtue of (52), the asymptotic stability of $x_{a}$ is now proven by studying the following system on $t \in[T,+\infty[$ :

$$
\left\{\begin{aligned}
\dot{q}_{a} & =\frac{1}{2} E\left(q_{a}\right)\left(\omega_{a}^{b e}+\Delta_{q}\right) \\
\dot{\omega}_{a}^{b e} & =-k_{a} q_{a v}-k(t) \omega_{a}^{b e}+J_{m b}^{-1} \Delta_{\omega} \\
\dot{z}_{a} & =A_{z} z_{a}+B_{1, z} \omega_{a}^{b e}
\end{aligned}\right.
$$

To this aim, we consider the following function:

$$
V_{a}\left(t, x_{a}\right)=k_{a}\left[\left(1-q_{a 0}\right)^{2}+\left|q_{a v}\right|^{2}\right]+\frac{1}{2}\left|\omega_{a}^{b e}\right|^{2}+\frac{\varepsilon}{2} z_{a}^{T} P_{z} z_{a}+\mu q_{a v}^{T} \omega_{a}^{b e}
$$


where $\varepsilon$ is a small positive constant to be clarified next and $\mu>0$ is a tuning parameter. From a simple completion of squares:

$$
-\frac{\mu}{2}\left|q_{a v}\right|^{2}-\frac{\mu}{2}\left|\omega_{a}^{b e}\right|^{2} \leq \mu q_{a v}^{T} \omega_{a}^{b e} \leq \frac{\mu}{2}\left|q_{a v}\right|^{2}+\frac{\mu}{2}\left|\omega_{a}^{b e}\right|^{2}
$$

it is readily checked that $V_{a}$ is a Lyapunov candidate function since it verifies:

$$
k_{a}\left(1-q_{a 0}\right)^{2}+\frac{k_{a}}{2}\left|q_{a v}\right|^{2}+\frac{1}{4}\left|\omega_{a}^{b e}\right|^{2}+\frac{\varepsilon}{2} z_{a}^{T} P_{z} z_{a} \leq V_{a} \leq k_{a}\left(1-q_{a 0}\right)^{2}+\frac{3 k_{a}}{2}\left|q_{a v}\right|^{2}+\frac{3}{4}\left|\omega_{a}^{b e}\right|^{2}+\frac{\varepsilon}{2} z_{a}^{T} P_{z} z_{a}
$$

when $0<\mu \leq \min \left\{k_{a}, \frac{1}{2}\right\}$.

Next, differentiating $V_{a}$ with respect to time and using (60) yields $\forall t \geq T$

$$
\begin{aligned}
\dot{V}_{a}= & k_{a} q_{a v}^{T}\left(\omega_{a}^{b e}+\Delta_{q}\right)+\left(\omega_{a}^{b e}+\mu q_{a v}\right)^{T}\left(-k_{a} q_{a v}-k(t) \omega_{a}^{b e}+J_{m b}^{-1} \Delta_{\omega}\right)-\varepsilon z_{a}^{T} Q_{z} z_{a} \\
& +\varepsilon z_{a}^{T} P_{z} B_{1, z} \omega_{a}^{b e}+\frac{\mu}{2}\left(\left(q_{a 0} I_{3}+S\left(q_{a v}\right)\right) \omega_{a}^{b e}\right)^{T} \omega_{a}^{b e} \\
\leq & -\mu k_{a}\left|q_{a v}\right|^{2}-k(t)\left|\omega_{a}^{b e}\right|^{2}-\varepsilon z_{a}^{T} Q_{z} z_{a}-\mu k(t) q_{a v}^{T} \omega_{a}^{b e} \\
& +\varepsilon z_{a}^{T} P_{z} B_{1, z} \omega_{a}^{b e}+\frac{\mu}{2}\left|\omega_{a}^{b e}\right|^{2}+k_{a} q_{a v}^{T} \Delta_{q}+\left(\omega_{a}^{b e}+\mu q_{a v}\right)^{T} J_{m b}^{-1} \Delta_{\omega}
\end{aligned}
$$

Considering equations (62) and (63), it is easily checked that

$$
\forall t \geq T, \quad k_{\min } \leq k(t) \leq k_{\max }
$$

with

$$
k_{\min }=k_{O}+\frac{2}{\bar{\omega}-\underline{\omega}}\left|J_{m b}^{-1}\right| \bar{d}, \quad k_{\max }=k_{O}+\frac{4}{\bar{\omega}-\underline{\omega}}\left|J_{m b}^{-1}\right| \bar{d}
$$

Combining (75) with (76) yields $\forall t \geq T$ :

$$
\begin{aligned}
\dot{V}_{a} \leq & -\mu k_{a}\left|q_{a v}\right|^{2}-k_{\min }\left|\omega_{a}^{b e}\right|^{2}-\varepsilon z_{a}^{T} Q_{z} z_{a}+\mu k_{\max }\left|q_{a v}\right|\left|\omega_{a}^{b e}\right| \\
& +\varepsilon z_{a}^{T} P_{z} B_{1, z} \omega_{a}^{b e}+\frac{\mu}{2}\left|\omega_{a}^{b e}\right|^{2}+k_{a} q_{a v}^{T} \Delta_{q}+\left(\omega_{a}^{b e}+\mu q_{a v}\right)^{T} J_{m b}^{-1} \Delta_{\omega} \\
\leq & -x_{a}^{T} Q_{a} x_{a}+k_{a} q_{a v}^{T} \Delta_{q}+\left(\omega_{a}^{b e}+\mu q_{a v}\right)^{T} J_{m b}^{-1} \Delta_{\omega}
\end{aligned}
$$

where:

$$
Q_{a}=\left[\begin{array}{ccc}
\mu k_{a} & \frac{\mu k_{\max }}{2} & O \\
\frac{\mu k_{\max }}{2} & k_{\min }-\frac{\mu}{2} & -\frac{\varepsilon}{2} P_{z} B_{1, z} \\
O & -\frac{\varepsilon}{2} P_{z} B_{1, z} & \varepsilon Q_{z}
\end{array}\right]
$$

which is positive definite for small values of $\mu$ and $\varepsilon>0$.

Finally, it is convenient to bound the coupling terms between the $\tilde{x}$ and $x_{a}$ subsystems, which is the scope of the following lemma (its proof is detailed in appendix B)

Lemma 5: there exists $\lambda>0$ such that $\forall t \geq T$

$$
k_{a} q_{a v}^{T} \Delta_{q}+\left(\omega_{a}^{b e}+\mu q_{a v}\right)^{T} J_{m b}^{-1} \Delta_{\omega} \leq \lambda\left|x_{a}\right|\left(|\tilde{x}|+\left|e_{z}\right|+\left|\omega_{r}\right|\right)
$$


Consequently, $\forall t \geq T$

$$
\dot{V}_{a} \leq-x_{a}^{T} Q_{a} x_{a}+\lambda\left|x_{a}\right|\left(|\tilde{x}|+\left|e_{z}\right|+\left|\omega_{r}\right|\right)
$$

$\omega_{r}$ (resp. $\tilde{x}$ and $e_{z}$ ) being in $\mathcal{L}_{2}$ from Assumption (A4) (resp. from the proof of part 1), it readily follows from the application of Barbalat's Lemma that $x_{a}$ asymptotically converges to the origin. The proof is now completed.

\section{Application to a flexible spacecraft attitude control problem}

A realistic application to a flexible spacecraft attitude control problem of the proposed design methodology is now presented.

\subsection{Numerical data}

In this application, the spacecraft model described in subsection 2.2 is considered with the following numerical data (borrowed from $\mathrm{Hu}(2009))$ :

$$
J_{m b}=\left[\begin{array}{ccc}
350 & 3 & 4 \\
3 & 270 & 10 \\
4 & 10 & 190
\end{array}\right] k g \cdot m^{2} \quad, \quad \delta=\left[\begin{array}{ccc}
6.46 & 1.28 & 2.16 \\
-1.26 & 0.92 & -1.67 \\
1.12 & 2.49 & -0.84
\end{array}\right] \mathrm{kg}^{1 / 2} \cdot m . s^{-2}
$$

The characteristics of the three flexible modes are summarized below in Table 1.

\begin{tabular}{|c|c|c|}
\hline & pulsation & damping ratio \\
\hline Flexible mode \#1 & $\omega_{1}=0.77 \mathrm{rad} / \mathrm{s}$ & $\xi_{1}=0.0056$ \\
\hline Flexible mode \#2 & $\omega_{2}=1.10 \mathrm{rad} / \mathrm{s}$ & $\xi_{2}=0.0086$ \\
\hline Flexible mode \#3 & $\omega_{3}=1.87 \mathrm{rad} / \mathrm{s}$ & $\xi_{3}=0.0130$ \\
\hline
\end{tabular}

Table 1.: Characteristics of the flexible modes

and the angular velocity constraints are as follows:

$$
\bar{\omega}=-\underline{\omega}=\left[\begin{array}{lll}
6 & 15 & 10
\end{array}\right]^{T} \mathrm{deg} / \mathrm{s}
$$

\subsection{Scenario}

The simulation starts from the initial state below:

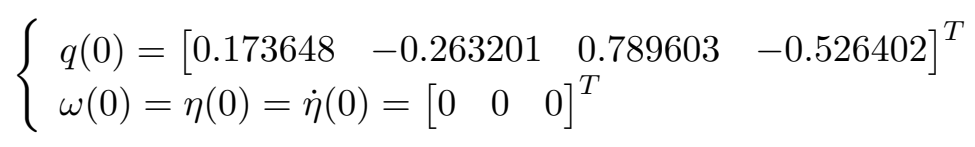

The flexible satellite attitude shall track a reference signal $q_{r}(t)$ defined by its initial value $q_{r}(0)=\left[\begin{array}{llll}1 & 0 & 0 & 0\end{array}\right]^{T}$ and its time-domain variation $\omega_{r}$ generated by a two-steps response of a first order filter $F_{r}(s)=(1+0.3 s)^{-1}$.

Moreover, a periodic disturbance torque is applied to the satellite:

$$
d(t)=\left[\begin{array}{lll}
0.3 \cos (0.01 t)+0.1 & 0.15 \sin (0.02 t)+0.3 \cos (0.025 t) \quad 0.3 \sin (0.01 t)+0.1
\end{array}\right]^{T} N m
$$


In our interval observer design, we will then use the bound $\bar{d}=\left[\begin{array}{lll}0.5 & 0.5 & 0.5\end{array}\right]^{T} \mathrm{Nm}$.

\subsection{Controller gains tuning}

For comparison purpose, we consider the nominal (state-feedback) control law resulting from Lemma 1 and the enhanced (output-feedback) version resulting from Theorem 1:

- the nominal control law gains are simply chosen by poles placement of the following system:

$$
\begin{aligned}
\dot{q}_{e v} & =\omega_{e} \\
J_{m b} \dot{\omega}_{e} & =-k_{p} q_{e v}-k_{d} \omega_{e}
\end{aligned}
$$

which simply consists of a linearization of the rigid part of the closed loop system (3a)-(3c)(11). Here, a satisfying pole placement was obtained for:

$$
k_{p}=0.25 J_{m b}, \quad k_{d}=0.1 J_{m b}
$$

- the enhanced control law is tuned by choosing the gains $k_{O}, k_{a}>0$ which must satisfy inequalities (65). Given $\bar{d}, \bar{\omega}, \underline{\omega}$, we selected $k_{a}=2$ in order to obtain $k_{O} \cong k_{d}(1,1)$.

\subsection{Nonlinear simulations results}

The spacecraft model with both nominal and enhanced control laws has been implemented in SimULINK $^{T M}$ files. Following the scenario presented in subsection 6.2, nonlinear simulations have been performed on a time range from 0 to $120 \mathrm{~s}$. The results are presented below on Figures 1,2 and 3 .

As is clarified in the paper, the use of an interval observer of the unmeasured disturbance torque is essential in our approach to enforce the angular rates constraints thanks to the OISTeR methodology. The behavior of this observer is illustrated by Figure 1. The tightness of the computed bounds as a function of time is clearly visualized.

Next, the angular velocities on the three axes are displayed in Figure 2 where a comparison between the nominal and enhanced controllers is proposed. As expected, every time a step input is applied, the angular velocities exceed the constraints on each axis when the nominal controller is used. This is however no longer the case with the enhanced control system. In this case indeed, the velocities are always kept in the prescribed limits.

The comparison of the nominal and enhanced controllers is further investigated in Figure 3.

- The first subplot (upper) illustrates the time-domain evolution of the tracking error (via the norm of the quaternion error $\left.\left\|q_{e v}(t)\right\|\right)$. It is easily observed that the nominal controller outperforms the OISTeR-based solution. But this is not surprising since velocities constraints are not respected in the first case. More precisely, when comparing both plots, the price to pay for constraints satisfaction is clearly visualized between $t \cong 50$ s and $t \cong 65 \mathrm{~s}$. During this time interval the reference quaternion is no longer tracked by the enhanced system. Next, the error starts to decrease as soon as the anti-windup loop becomes active.

- The second subplot (middle) illustrates the activity of the flexible mode. Quite interestingly, it is observed that the excitation of the flexible modes is considerably lower when the enhanced controller is used. Here again, this is not surprising since the angular velocities are now controlled.

- The last and third subplot (lower) illustrates the control activity which, as expected also, is significantly lower when the OISTeR-based system is used. 


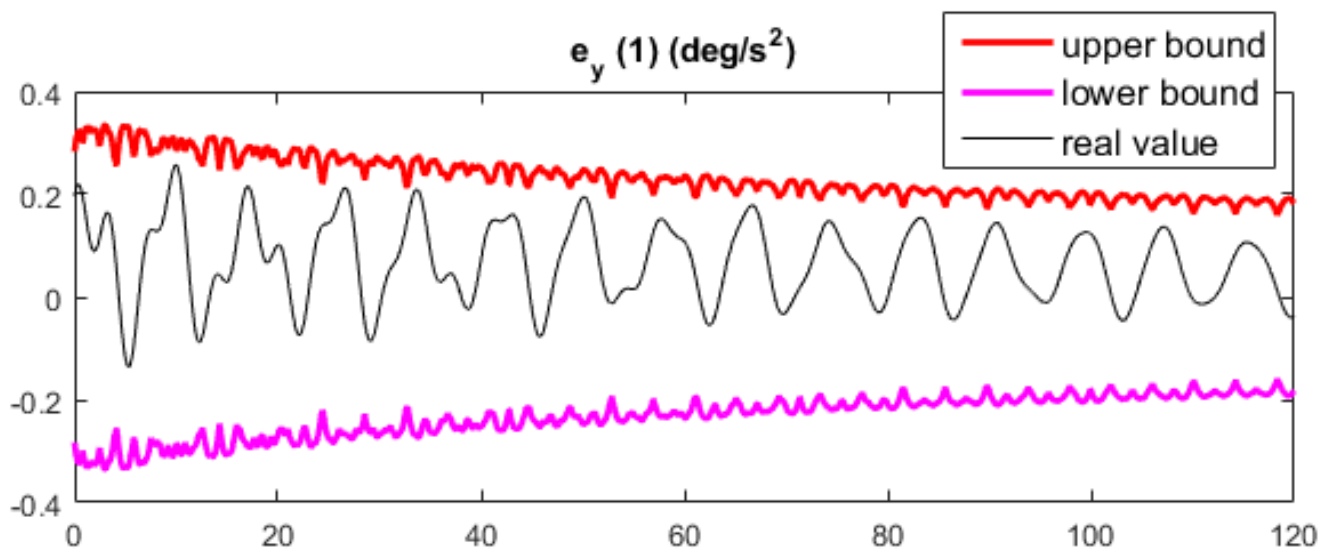

$e_{y}(2)\left(d e g / s^{2}\right)$

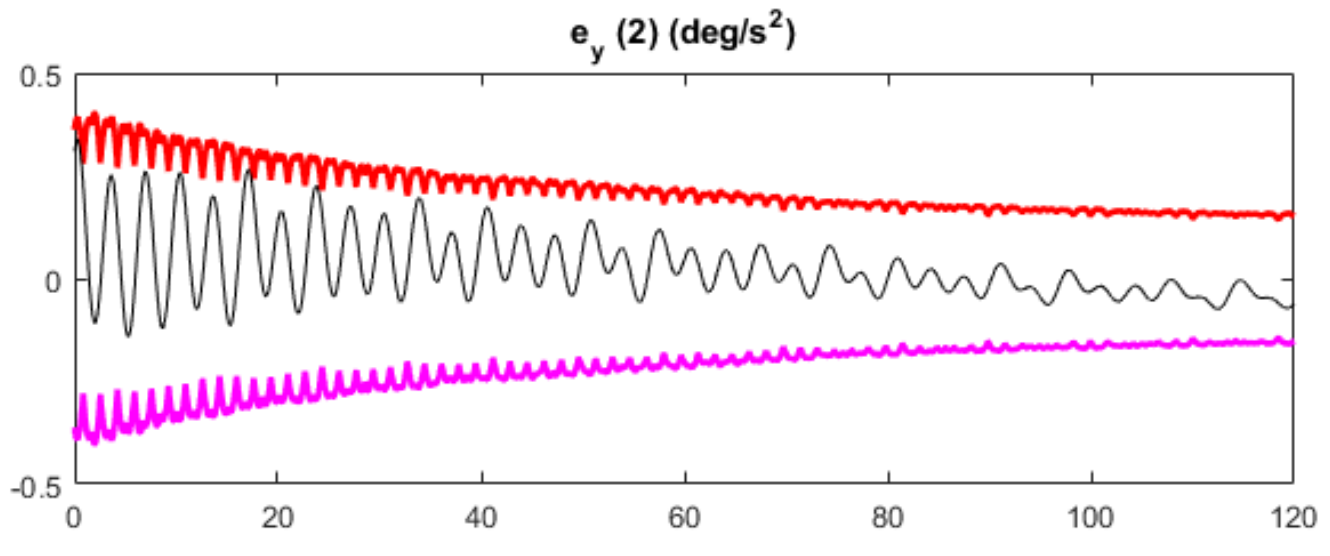

$e_{y}(3)\left(d e g / s^{2}\right)$

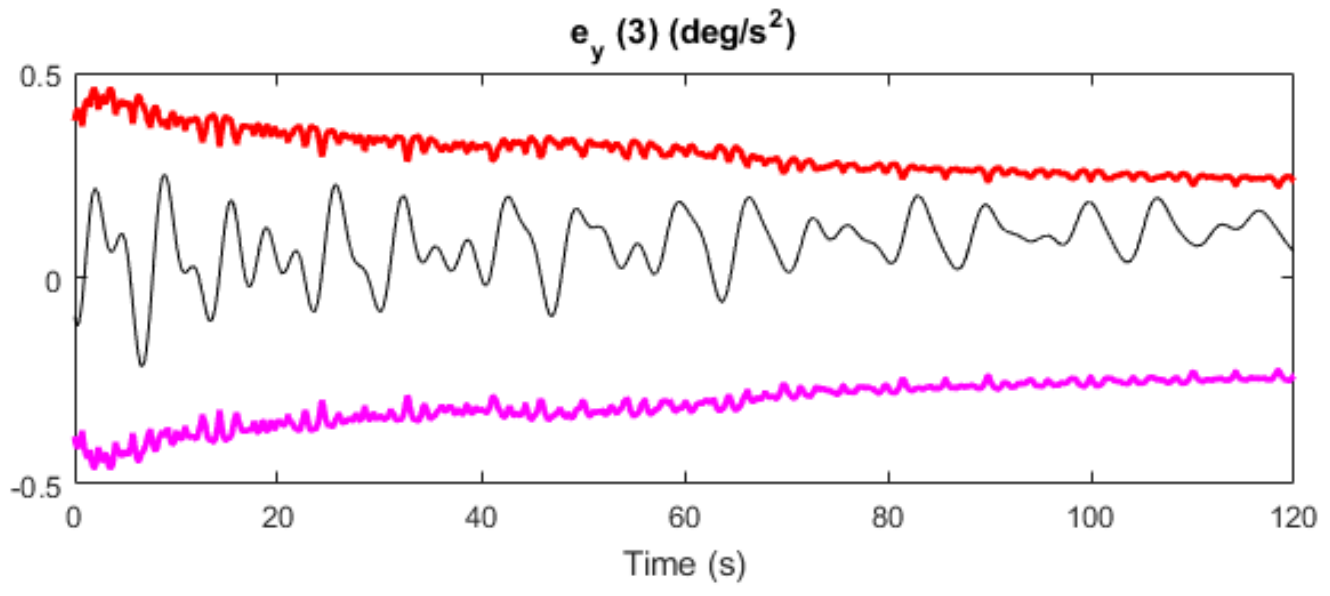

Figure 1.: Interval observer (bounds detection on the unmeasured disturbance torque $e_{y}$ ) 

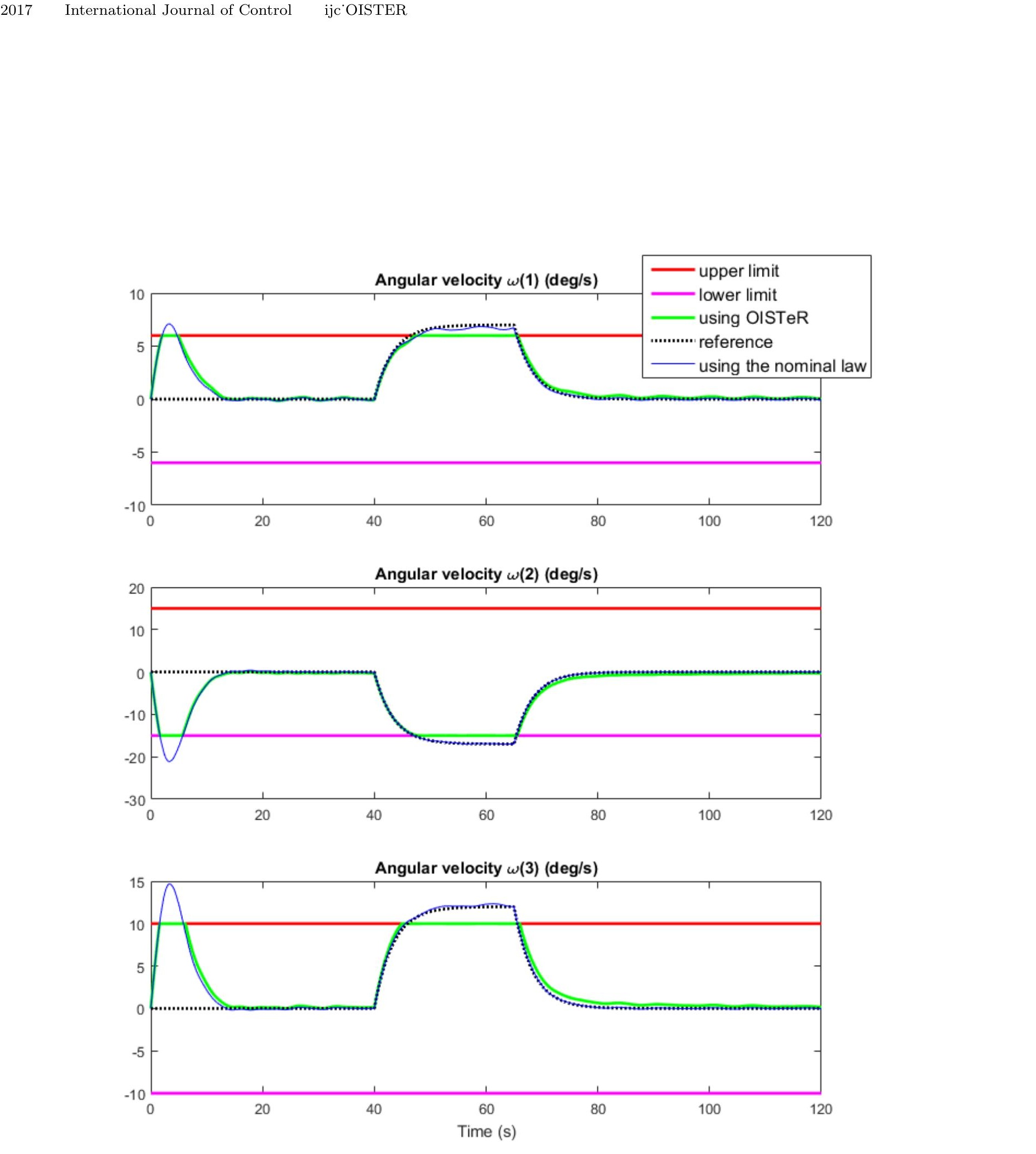

Figure 2.: Comparison of the angular velocities on each axis with the nominal and enhanced (OISTeR-based) control systems

Time (s)

$$
\text { so }
$$$$
\text { (1) }
$$

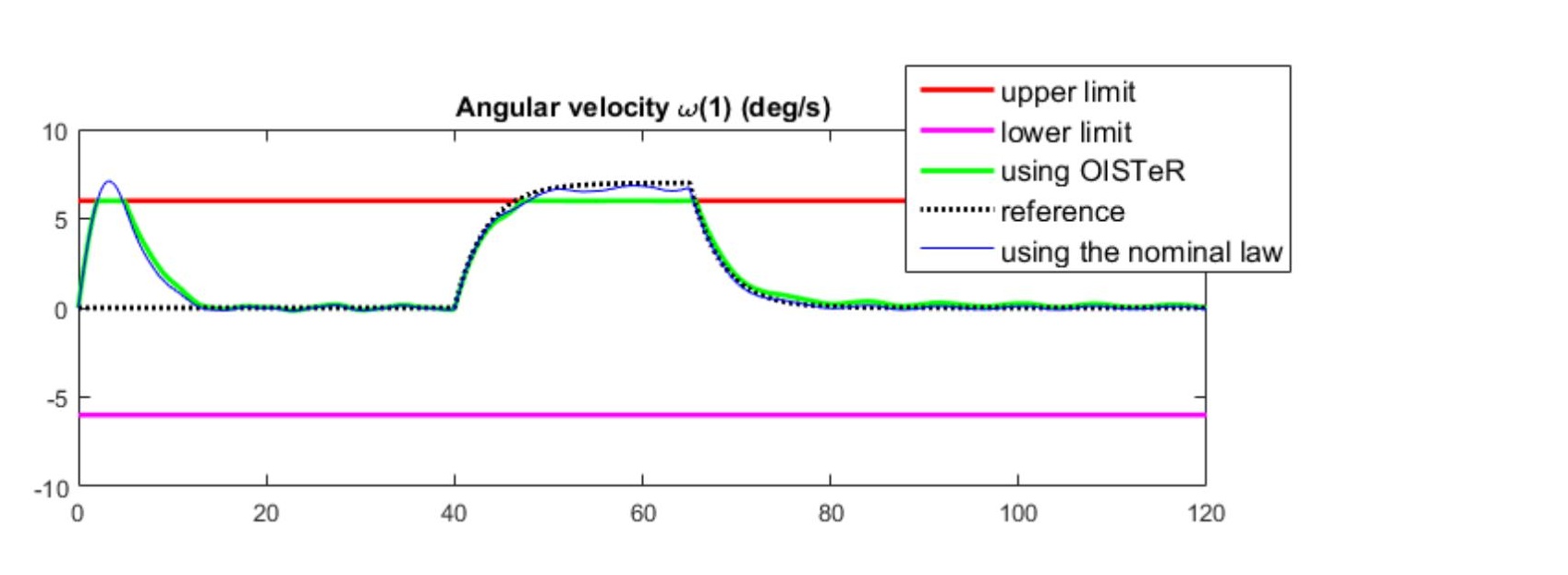

(1)
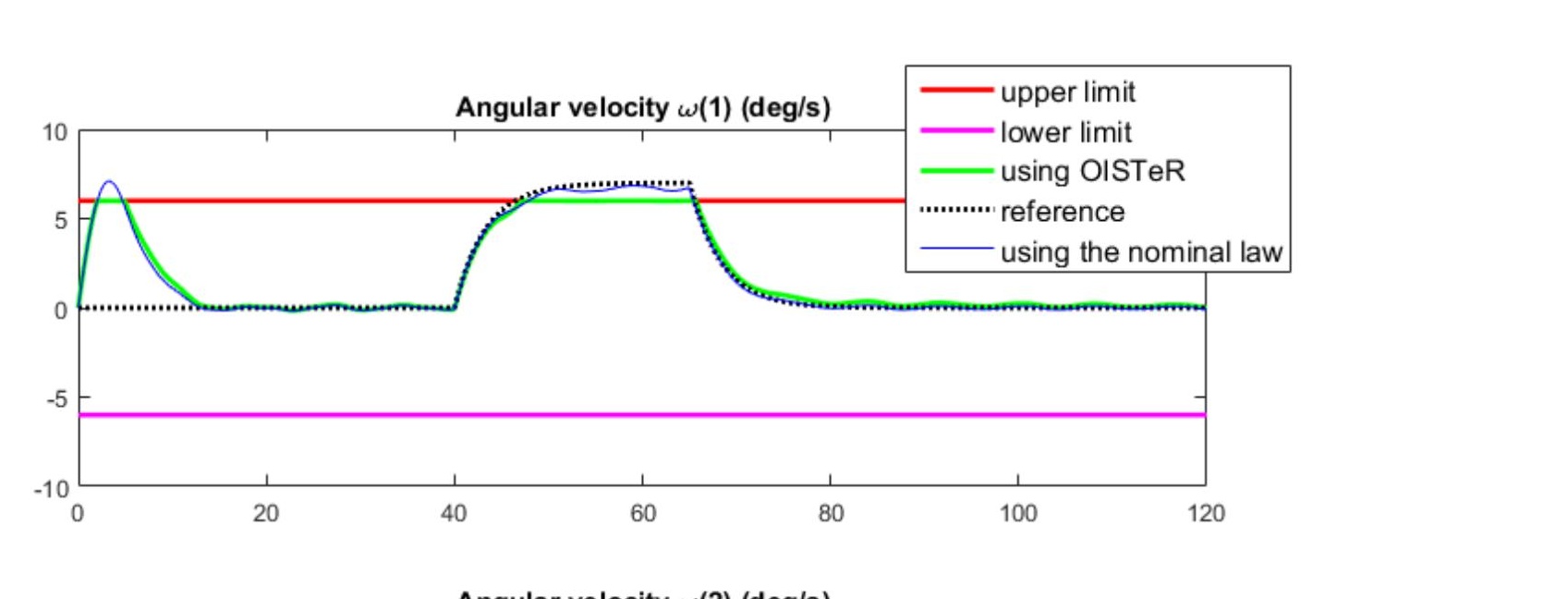

\begin{abstract}
(OTSTeR-based) control systems
\end{abstract}
s.

(1)



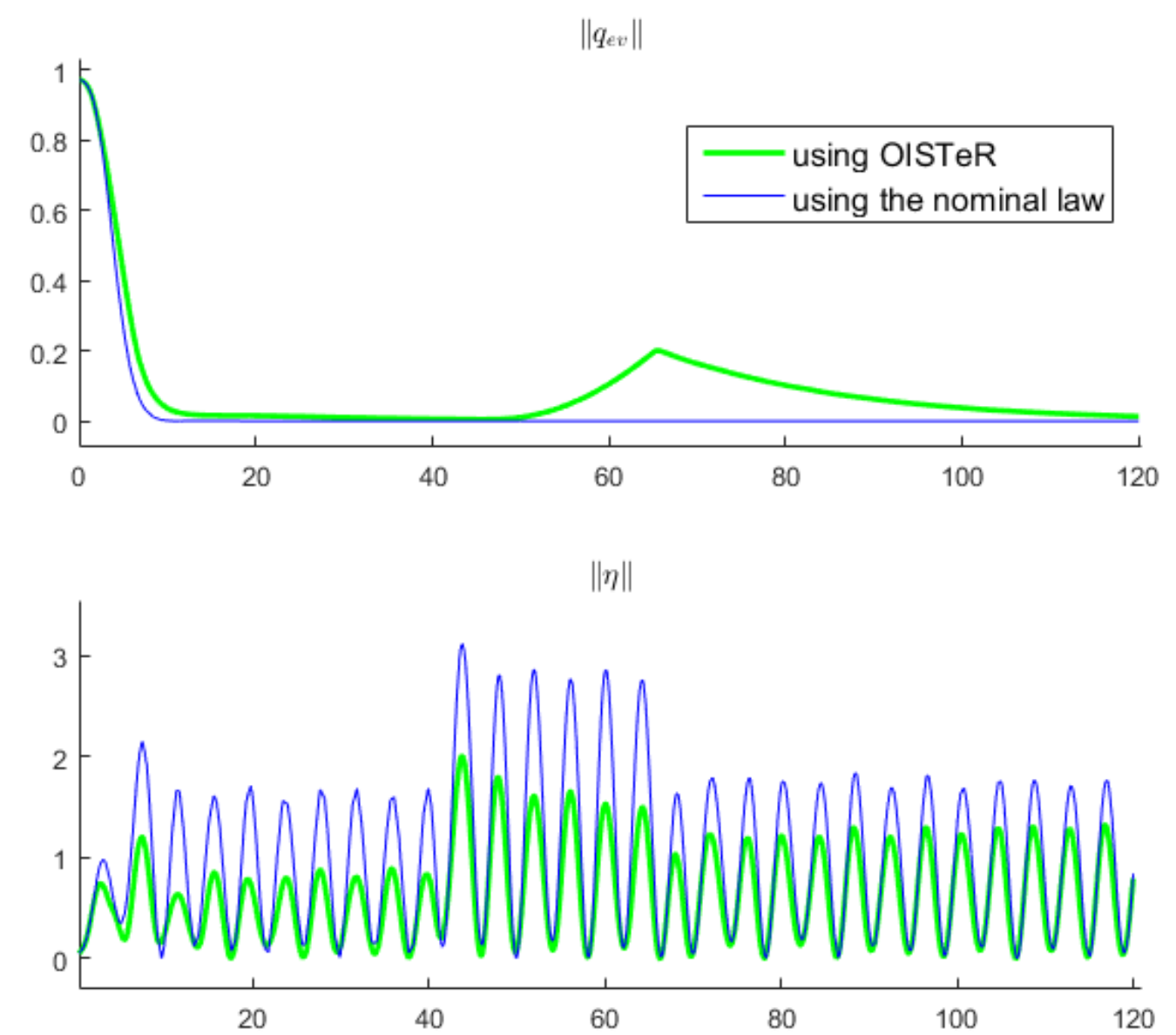

$\|u\|(\mathrm{Nm})$

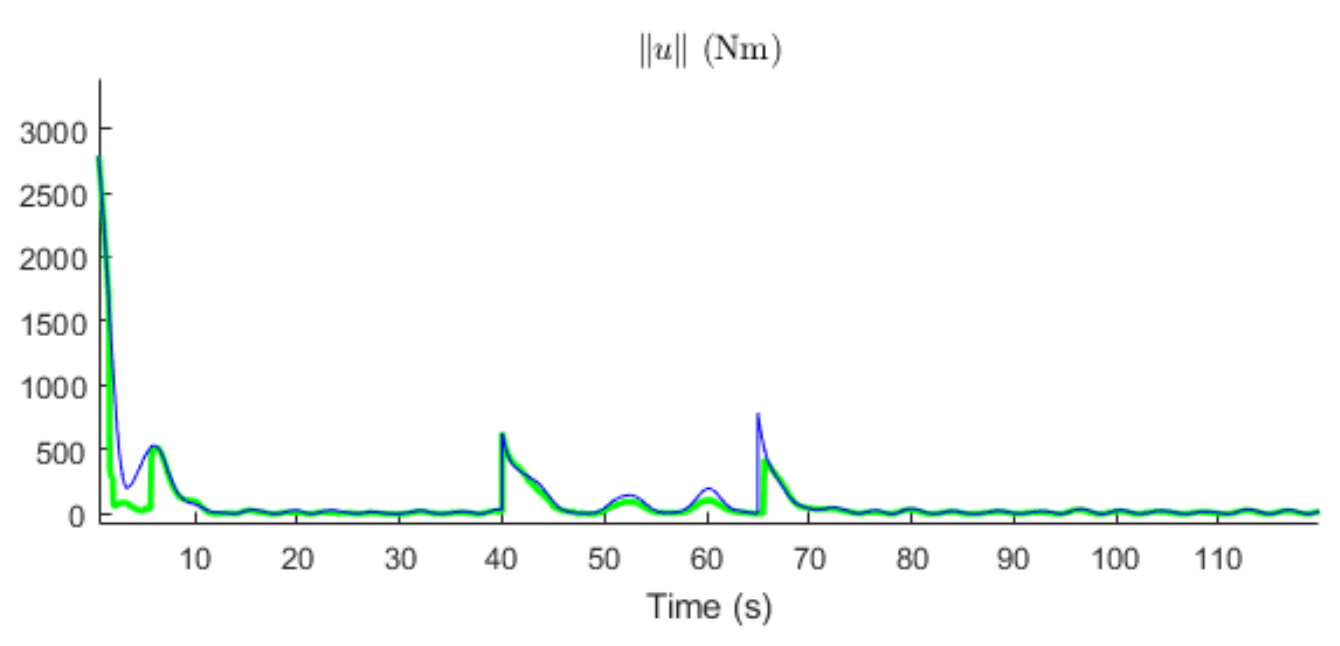

Figure 3.: Further comparison of the nominal and enhanced controllers through the tracking error $\left(\left\|q_{e v}(t)\right\|\right)$, the activity of the flexible modes $(\|\eta(t)\|)$ and the control effort $(\|u(t)\|)$. 


\section{Conclusions}

The problem of output-feedback trajectory tracking of flexible spacecraft attitude under angular velocity constraints has been addressed in this paper. Given a nominal controller which solves the tracking problem in the full state-feedback case without velocity constraints, it is proven that the general problem can be solved by saturating the nominal controller by an Output to Input Saturation. Such a saturated control law is then applied in combination with a nonlinear antiwindup loop. Note that one key feature of this result is to use an interval observer not only to estimate the flexible modes but also to provide at each instant their lower and upper bounds, which is crucial to ultimately guarantee the satisfaction of the velocity constraints. In future research, we plan to extend this work to handle parametric uncertainties and control torque limitations.

\section{References}

Akella, M., Thakur, D., \& Mazenc, F. (2015). Partial Lyapunov strictification: Smooth angular velocity observers for attitude tracking control. Journal of Guidance, Control, and Dynamics, 38(3), 442-451.

Burlion, L. (2012). A new saturation function to convert an output constraint into an input constraint. In 20th Mediterranean Conference on Control Automation (MED) (p. 1217-1222).

Chambon, E., Burlion, L., \& Apkarian, P. (2015a). Output to input saturation transformation: Demonstration and application to disturbed linear systems. In IEEE 54th Annual Conference on Decision and Control (CDC) (p. 7566-7571).

Chambon, E., Burlion, L., \& Apkarian, P. (2015b). Robust output interval constraint using O/I saturation transformation with application to uncertain linear launch vehicle. In European Control Conference (ECC) (p. 1802-1807).

Chen, Z., \& Huang, J. (2009). Attitude tracking and disturbance rejection of rigid spacecraft by adaptive control. IEEE Transactions on Automatic Control, 54(3), 600-605.

Di Gennaro, S. (2002). Output attitude tracking for flexible spacecraft. Automatica, 38(10), 1719 - 1726.

Galeani, S., Tarbouriech, S., Turner, M., \& Zaccarian, L. (2009). A tutorial on modern anti-windup design. European Journal of Control, 15(34), 418 - 440.

G.C. Goodwin, M. S., \& de Dona, J. (2004). Constrained control and estimation: an optimization approach. Springer.

Gennaro, S. D. (2003). Output stabilization of flexible spacecraft with active vibration suppression. IEEE Transactions on Aerospace and Electronic Systems, 39(3), 747-759.

Glattfelder, A., \& Schaufelberger, W. (2003). Control systems with input and output constraints. Springer.

Gupta, R., Kalabic, U. V., Cairano, S. D., Bloch, A. M., \& Kolmanovsky, I. V. (2015, July). Constrained spacecraft attitude control on $\mathrm{SO}(3)$ using fast nonlinear model predictive control. In American Control Conference (ACC) (p. 2980-2986).

Hegrenaes, O., Gravdahl, J., \& Tondel, P. (2005). Spacecraft attitude control using explicit model predictive control. Automatica, 41(12), 2107-2114.

H.-H. Zhang, F. W., \& Trivailo., P. (2008). Spin-axis stabilisation of underactuated rigid spacecraft under sinusoidal disturbance. International Journal of Control, 81(12), 1901-1909.

$\mathrm{Hu}$, Q. (2009). Robust adaptive backstepping attitude and vibration control with $L_{2}$-gain performance for flexible spacecraft under angular velocity constraint. Journal of Sound and Vibration, 327(35), 285 298.

Lovera, M., \& Astolfi, A. (2004). Spacecraft attitude control using magnetic actuators. Automatica, 40(8), $1405-1414$.

Luzi, A.-R., Peaucelle, D., Biannic, J.-M., Pittet, C., \& Mignot, J. (2014). Structured adaptive attitude control of a satellite. International Journal of Adaptive Control and Signal Processing, 28(7-8), 664685 .

Mazenc, F., \& Bernard, O. (2010, Feb). Asymptotically stable interval observers for planar systems with complex poles. IEEE Transactions on Automatic Control, 55(2), 523-527.

Tarbouriech, S., \& Turner, M. (2009). Anti-windup design: an overview of some recent advances and open problems. Control Theory Applications, IET, 3(1), 1-19. 
Tayebi, A. (2008). Unit quaternion-based output feedback for the attitude tracking problem. IEEE Transactions on Automatic Control, 53(6), 1516-1520.

Trégouët, J.-F., Arzelier, D., Peaucelle, D., Pittet, C., \& Zaccarian, L. (2015). Reaction wheels desaturation using magnetorquers and static input allocation. IEEE Transactions on Control Systems Technology, $23(2), 525-539$.

Zhang, X., \& Cheng, D. (2012). Nonlinear internal model based attitude tracking and disturbance rejection. Asian Journal of Control, 14(5), 1397-1402.

\section{Appendix A. Proof of Lemma 2}

First of all, let us introduce the following notation. Note that this notation (which is very specific and merely used in this proof) was not given before to avoid breaking the flow of the paper derivations.

Notation 4: we introduce the following two functions (as in Mazenc and Bernard (2010)):

$$
\mathcal{L}(s)=\max \{0, s\}, \quad \mathcal{M}(s)=\min \{0, s\}
$$

Then, given a time-varying matrix $\Pi(t)$, we define:

$$
\Pi_{p}(t)=\left(\mathcal{L}\left(\Pi_{i, j}(t)\right)\right), \quad \Pi_{m}(t)=\left(\mathcal{M}\left(\Pi_{i, j}(t)\right)\right),
$$

As a consequence of $|s|=\mathcal{L}(s)-\mathcal{M}(s)$, one has $|\Pi(t)|=\Pi_{p}(t)-\Pi_{m}(t)$, such an equality being understood element-wise.

Let us now prove Lemma 2:

Proof. First, let us consider:

$$
\dot{e}_{z}=A_{z} e_{z}
$$

where $\left|e_{z, 0}\right| \leq \frac{z_{0}^{+}-z_{0}^{-}}{2}$.

Let us note $\mathcal{N}=\left[N_{1}^{T}, \ldots, N_{N}^{T}\right]^{T}$ where

$$
N_{i}=\left[e_{z, i}, e_{z, i+N}\right]^{T} \quad, \forall i \in[1, N]
$$

These relations define a change of coordinates which is now denoted as follows:

$$
\mathcal{N}=P e_{z}
$$

It is readily seen that the $N_{i}$ 's verify:

$$
\dot{N}_{i}=J_{i} N_{i} \quad, i \in[1, N]
$$

where

$$
J_{i}=\left[\begin{array}{cc}
0 & 1 \\
-\omega_{i}^{2} & -2 \xi_{i} \omega_{i}
\end{array}\right]
$$


Following Mazenc and Bernard (2010), we apply the change of coordinate $\zeta_{i}=\lambda_{i}(t) N_{i}$ where

$$
\lambda_{i}(t)=\left[\begin{array}{cc}
\cos \left(\omega_{i} t\right)-\xi_{i} \sin \left(\omega_{i} t\right) & -\frac{1}{\omega_{i}} \sin \left(\omega_{i} t\right) \\
\omega_{i} \sin \left(\omega_{i} t\right) & \cos \left(\omega_{i} t\right)+\xi_{i} \sin \left(\omega_{i} t\right)
\end{array}\right]
$$

which gives:

$$
\dot{\zeta}_{i}=-\kappa_{i} \zeta_{i} \quad, i \in[1, N]
$$

where $\kappa_{i}=\xi_{i} \omega_{i}$.

(Note that the change of coordinate is well defined ; indeed, $\operatorname{det}\left(\lambda_{i}(t)\right)=\cos ^{2}\left(\omega_{i} t\right)+(1-$ $\left.\xi_{i}^{2}\right) \sin ^{2}\left(\omega_{i} t\right) \neq 0$ since the damping ratios are assumed to satisfy $\left.0<\xi_{i}<0\right)$

Finally, noting $M=\operatorname{diag}\left(\left\{-\kappa_{i} I_{2}, i=1 . . N\right\}\right)$ and $\lambda(t)=\operatorname{diag}\left(\left\{\lambda_{i}(t), i=1 . . N\right\}\right)$, system (A3) is equivalent to:

$$
\begin{aligned}
& \dot{\zeta}=M \zeta \\
& \zeta=\lambda(t) P e_{z}:=Q(t) e_{z}
\end{aligned}
$$

It follows from $\left|e_{z, 0}\right| \leq \frac{z_{0}^{+}-z_{0}^{-}}{2}$ and from (A9b) that

$$
\zeta(0) \in\left[\zeta_{0}^{-}, \zeta_{0}^{+}\right]
$$

where

$$
\left\{\begin{array}{l}
\zeta_{0}^{+}=Q_{p}(0) \frac{z_{0}^{+}-z_{0}^{-}}{2}-Q_{m}(0) \frac{z_{0}^{+}-z_{0}^{-}}{2}=|Q(0)| \frac{z_{0}^{+}-z_{0}^{-}}{2} \\
\zeta_{0}^{-}=Q_{m}(0) \frac{z_{0}^{+}-z_{0}^{-}}{2}-Q_{p}(0) \frac{z_{0}^{+}-z_{0}^{-}}{2}=-|Q(0)| \frac{z_{0}^{+}-z_{0}^{-}}{2}
\end{array}\right.
$$

$M$ being Hurwitz Metzler, the following system defines an interval observer for system (A9a)

$$
\left\{\begin{array}{l}
\dot{\zeta}^{+}=M \zeta^{+} \\
\dot{\zeta}^{-}=M \zeta^{-}
\end{array}\right.
$$

where $\zeta^{+}(0)=\zeta_{0}^{+}\left(\operatorname{resp} \cdot \zeta^{-}(0)=\zeta_{0}^{-}\right)$.

Finally, such an interval observer can be used to bound the output $e_{y}$ since:

$$
\begin{aligned}
e_{y} & =J_{m b}^{-1}\left(-S(\omega) G_{z} e_{z}+C_{z} e_{z}+d\right) \\
& =J_{m b}^{-1}\left(\left(-S(\omega) G_{z}+C_{z}\right) Q(t)^{-1} \zeta+d\right) \\
& :=\Pi(t, \omega) \zeta+J_{m b}^{-1} d
\end{aligned}
$$

Indeed, it is readily seen that $e_{y} \in\left[e_{y}^{-}, e_{y}^{+}\right]$where:

$$
\left\{\begin{aligned}
e_{y}^{+}: & :=\Pi_{p}(t, \omega) \zeta^{+}+\Pi_{m}(t, \omega) \zeta^{-}+\left|J_{m b}^{-1}\right| \bar{d} \\
e_{y}^{-} & :=\Pi_{m}(t, \omega) \zeta^{+}+\Pi_{p}(t, \omega) \zeta^{-}-\left|J_{m b}^{-1}\right| \bar{d}
\end{aligned}\right.
$$

which simplifies as follows:

$$
\left\{\begin{array}{l}
e_{y}^{+}:=|\Pi(t, \omega)| \zeta^{+}+\left|J_{m b}^{-1}\right| \bar{d} \\
e_{y}^{-}:=-e_{y}^{+}
\end{array}\right.
$$


Indeed, it readily follows from (A11)-(A12) that $\forall t, \zeta^{+}(t)=-\zeta^{-}(t)$. The proof of the Lemma is then completed.

\section{Appendix B. Proof of Lemma 5}

The proof of the Lemma readily follows from the proofs of the two following inequalities:

(1) there exists $\lambda_{4}, \lambda_{5}, \lambda_{6}>0$ such that $\forall t \geq T$ :

$$
\left(\omega_{a}^{b}+\mu q_{a v}\right)^{T} \Delta_{\omega} \leq \lambda_{4}\left|x_{a}\right||\tilde{x}|+\lambda_{5}\left|x_{a}\right|\left|e_{z}\right|+\lambda_{6}\left|x_{a}\right|\left|\omega_{r}\right|
$$

(2) there exists $\lambda_{7}>0$ such that

$$
q_{a v}^{T} \Delta_{q} \leq \lambda_{7}\left|x_{a}\right||\tilde{x}|
$$

\section{B.1 Part 1}

Starting from equation (56), we obtain (B4) by using (11)-(44a)-(44c)-(58) :

$$
\begin{aligned}
\left(\omega_{a}^{b}+\mu q_{a v}\right)^{T} \Delta_{\omega}= & \left(\omega_{a}^{b}+\mu q_{a v}\right)^{T}\left(\operatorname{OISat}\left(u_{a}+\tilde{u}_{n}\right)-\operatorname{OISat}\left(u_{a}\right)-\tilde{u}_{n}+N\left(\tilde{\omega}+\omega_{r}^{b}, \tilde{\omega}, \hat{\tilde{z}}, \omega_{r}^{b}\right)\right. \\
& \left.+\left(N\left(\omega, \omega_{a}^{b e}, z_{a}, 0\right)-N\left(\omega, \omega_{e}, \hat{z}_{e}, \omega_{r}^{b}\right)\right)\right) \\
\leq & 3(1+\mu)\left|x_{a}\right|\left(\lambda_{\max }\left(J_{m b}\right)\left|\Delta_{S}\right|+|\tilde{\Delta}|\right)
\end{aligned}
$$

where

$$
\begin{gathered}
\Delta_{S}:=S a t_{k(t) \underline{\omega}+\bar{e}_{y}}^{k(t) \bar{\omega}-\bar{e}_{y}}(\alpha+k(t) \omega)-S a t_{k(t) \underline{\omega}+\bar{e}_{y}}^{k(t) \bar{\omega}-\bar{e}_{y}}\left(\alpha_{a}+k(t) \omega_{a}^{b e}\right) \\
\tilde{\Delta}:=\quad J_{m b}(-k(t) \tilde{\omega})-C_{z} \tilde{z}-D_{z} \tilde{\omega}-\tilde{u}_{n}-N\left(\omega, \tilde{\omega}, \hat{\tilde{z}}, \omega_{r}^{b}\right) \\
\quad-C_{z}\left(z_{r}-e_{z}\right)-\left(J_{m b} k(t)+D_{z}\right) \omega_{r}^{b}
\end{gathered}
$$

Looking at (44b)-(59), we observe that

$$
\begin{aligned}
\alpha+k(t) \omega= & \alpha_{a}+k(t) \omega_{a}^{b e}+k(t)\left(\tilde{\omega}+\omega_{r}^{b}\right)+J_{m b}^{-1}\left(N\left(\omega, \omega_{a}^{b e}, z_{a}, 0\right)-N(\omega, \omega, \hat{z}, 0)\right. \\
& \left.+\tilde{u}_{n}+C_{z}\left(\tilde{z}-e_{z}+z_{r}\right)+D_{z}\left(\tilde{\omega}+\omega_{r}\right)\right)
\end{aligned}
$$

Substituting (B7) in (B5) and using the fact that Sat is 1-Lipschitz yields

$$
\begin{aligned}
\left|\Delta_{S}\right| \leq & \mid k(t)\left(\tilde{\omega}+\omega_{r}^{b}\right)+J_{m b}^{-1}\left(N\left(\omega, \omega_{a}^{b e}, z_{a}, 0\right)-N(\omega, \omega, \hat{z}, 0)\right. \\
& \left.+\tilde{u}_{n}+C_{z}\left(\tilde{z}-e_{z}+z_{r}\right)+D_{z}\left(\tilde{\omega}+\omega_{r}\right)\right) \mid \\
\leq & \mid k(t)\left(\tilde{\omega}+\omega_{r}^{b}\right)+J_{m b}^{-1}\left(-N\left(\omega, \tilde{\omega}+\omega_{r}^{b}, \hat{\tilde{z}}+z_{r}, 0\right)\right. \\
& \left.+\tilde{u}_{n}+C_{z}\left(\tilde{z}-e_{z}+z_{r}\right)+D_{z}\left(\tilde{\omega}+\omega_{r}\right)\right) \mid
\end{aligned}
$$


Looking at the expressions (6)(resp. (11)-(44a)) of $N$ (resp. $\tilde{u}$ ) and bearing in mind the fact that $\omega$ is bounded and $k(t)$ is dominated by a constant $k_{\max }>0$ for $t \geq T$, it is not difficult to see that $\left|\Delta_{S}\right|$ (resp. $\left.|\tilde{\Delta}|\right)$ given by (B9)(resp. (B6) ) is Lipschitz with respect to the variables $\tilde{x}, e_{z}$ and $\omega_{r}$ for all $t \geq T$. The proof of (B1) readily follows from this fact plus the use of (B4).

\section{B.2 Part 2}

Starting from equation (54), one can successively prove that:

$$
\begin{aligned}
q_{a v}^{T} \Delta_{q} & =q_{a v}^{T}\left(R(\tilde{q})^{T}-I_{3}\right) \omega_{a}^{b e} \\
& =q_{a v}^{T}\left(\left(\tilde{q}_{0}^{2}-\tilde{q}_{v}^{T} \tilde{q}_{v}-1\right) I_{3}+2 \tilde{q}_{v} \tilde{q}_{v}^{T}-2 \tilde{q}_{0} S\left(\tilde{q}_{v}\right)\right) \omega_{a}^{b e} \\
& =q_{a v}^{T}\left(-2 \tilde{q}_{v}^{T} \tilde{q}_{v} I_{3}+2 \tilde{q}_{v} \tilde{q}_{v}^{T}-2 \tilde{q}_{0} S\left(\tilde{q}_{v}\right)\right) \omega_{a}^{b e} \\
& =q_{a v}^{T}\left(2 S\left(\tilde{q}_{v}\right)^{2}-2 \tilde{q}_{0} S\left(\tilde{q}_{v}\right)\right) \omega_{a}^{b e} \\
& \left.=2 q_{a v}^{T}\left(S\left(\tilde{q}_{v}\right)-\tilde{q}_{0} I_{3}\right)\right) S\left(\tilde{q}_{v}\right) \omega_{a}^{b e} \\
& \leq 2\left|q_{a v}\right|\left(\sqrt{2}\left|\tilde{q}_{v}\right|+1\right) \sqrt{2}\left|\tilde{q}_{v}\right|\left|\omega_{a}^{b e}\right| \\
& \leq 2(2+\sqrt{2})\left|\tilde{q}_{v}\right|\left|\omega_{a}^{b e}\right| \\
& \leq 8|\tilde{x}|\left|x_{a}\right|
\end{aligned}
$$

where (B10) is obtained by using the relation $S(x)^{2}=-\left(x^{T} x\right) I_{3}+x x^{T}$

where (B11) is obtained by using the relation $\left|S\left(q_{v}\right)\right| \leq \sqrt{2\left|q_{v}\right|^{2}}=\sqrt{2}\left|q_{v}\right|$ which readily follows from the well known relation $|A| \leq|A|_{F}=\sqrt{\operatorname{trace}\left(A^{T} A\right)}$ (substituting $A$ for $S\left(q_{v}\right)$ ).

The proof is thus completed for any $\lambda_{7} \geq 8$. 